\title{
Preliminary Study on the Relationship of BRAF Mutations with the Outcome of the First 131 I Radiotherapy and Malignant Biological Characteristics in Papillary Thyroid Carcinoma
}

\author{
Linjue Shangguan $\mathbb{D}^{1, *}$ \\ Peipei Zhang' \\ Shengwei Fang' \\ Kaili Xiang' \\ Yawen Geng' \\ Dingcun Luo $\mathbb{D}^{2, *}$ \\ Chunlei Zhaol,* \\ 'Department of Nuclear Medicine, \\ Hangzhou Cancer Hospital, Hangzhou, \\ 310002, People's Republic of China; \\ ${ }^{2}$ Department of Tumor Surgery, \\ Hangzhou First People's Hospital, \\ Hangzhou, 310006, People's Republic of \\ China
}

*These authors contributed equally to this work

\begin{abstract}
Objective: To investigate the relationship of $B R A F$ mutation with the outcome of the first postoperative ${ }^{131}$ I treatment and malignant biological characteristics in papillary thyroid carcinoma (PTC).
\end{abstract}

Methods: Thirty-three patients with PTC who underwent their first ${ }^{131}$ I treatment after total thyroidectomy were enrolled in this study. BRAF mutation in postoperative tumor tissue and circulating tumor DNA (ctDNA) in peripheral blood at the time of ${ }^{131} \mathrm{I}$ treatment were detected. According to the status of $B R A F$ mutation, all patients were divided into 2 groups in each category of tumor tissues and ctDNA, respectively: 1) $B R A F$ mutation, 2) $B R A F$ wild-type. The Fisher's exact test was performed to analyze the relationship of $B R A F$ mutation in either tumor tissue or ctDNA with the outcome of the first ${ }^{131}$ I treatment and malignant characteristics of PTC.

Results: $B R A F$ mutation was detected in tumor tissues in 25 patients $(25 / 33,75.8 \%)$, and all the patients had single mutation site. In ctDNA, BRAF mutation was detected in 5 patients $(5 / 33,15.2 \%)$, and all the patients had single mutation site. In both tumor tissues and ctDNA, $B R A F$ mutation showed no relationship with the outcome of first ${ }^{131} \mathrm{I}$ treatment and the malignant biological characteristics $(\mathrm{P}>0.05)$.

Conclusion: The value of $B R A F$ mutation alone might be limited in predicting therapeutic outcome of the first ${ }^{131}$ I treatment in PTC. No definitive relevance was found between $B R A F$ mutation and malignant biological features in PTC.

Keywords: gene mutation, $B R A F$, radiotherapy, papillary thyroid carcinoma

\section{Introduction}

Thyroid carcinoma is the most common malignancy of the endocrine system. PTC accounts for approximately $85 \%$ of thyroid carcinomas. ${ }^{1}$ Most PTCs are clinically indolent and usually have a favorable prognosis with a 15-year survival rate of more than $81 \%$ if treated with systemic comprehensive treatment consisting of complete surgical resection of the thyroid, post-operative ${ }^{131}$ I treatment and thyroid stimulating hormone (TSH) suppression with thyroxine (T4). Post-operative ${ }^{131} \mathrm{I}$ treatment is the most crucial adjuvant therapy that can significantly decrease recurrence of the disease in patients with PTC, resulting in an enhanced disease-free survival (DFS). However, the progression of the disease including distant metastasis, recurrence and resistance to 
${ }^{131}$ I treatment may occur in about $2-5 \%$ of PTC patients and lead to worse survival.

The understanding of genomic and molecular involvement in PTC has been profoundly enhanced in recent years. ${ }^{2,3}$ Among the gene mutations in PTC, BRAF mutation is the most frequent one, with an incidence of approximately $49 \%{ }^{4} B R A F$ mutation can greatly activate MAPK-pathway, subsequently promoting the proliferation and division of the cell independent of upstream signaling factors. Previous studies have found that $B R A F$ mutation was associated with malignant progression of PTC and may be useful as a molecular biomarker for predicting the prognosis of PTC. ${ }^{5-10}$

As a newly developed technology, liquid biopsy based on ctDNA has been widely used in the diagnosis and predicting prognosis in various tumors, such as lung cancer, breast cancer, colon cancer and anaplastic thyroid cancer. $^{11-13}$ ctDNA is single or double stranded DNA carrying the mutations of the original tumor released by tumor cells in the blood. Compared with traditional tissuebased biopsy, liquid biopsy has the advantages of monitoring the real-time status of mutation non-invasively with high sensitivity and specificity. ${ }^{14,15}$

So far, ctDNA detection has been increasingly applied in patients with thyroid cancer. Qin et al found that ctDNA is a valuable tool to detect the tumor molecular profile in anaplastic thyroid cancer (ATC). ${ }^{16}$ However, the clinical significance of gene mutations in ctDNA in PTC has not been understood profoundly. Considering that the patients with PTC after thyroidectomy are the largest population receiving ${ }^{131}$ I treatment, we carried out the present study to investigate the relationship of $B R A F$ mutation, in both ctDNA and tumor tissue, with the malignant biological characteristics and therapeutic outcome of first ${ }^{131} \mathrm{I}$ treatment in PTC patients.

\section{Materials and Methods}

\section{Patients and Samples}

Thirty-three PTC patients (after total thyroidectomy) who accepted the first postoperative ${ }^{131}$ I treatment in Hangzhou Cancer Hospital from April 2016 to July 2018 were enrolled in this study. All patients had moderate or high risk of recurrence of the disease according to the 2015 ATA guidelines. ${ }^{17}$ The inclusion criteria were defined as follows: i) total thyroidectomy was performed, ii) postoperative pathology was PTC, iii) low serum thyroglobulin antibody $(\mathrm{TgAb})$ level $(<100 \mathrm{kU} / \mathrm{L})$ (because serum thyroglobulin ( $\mathrm{Tg}$ ) level could be falsely lowered if TgAb level is high), iv) no ${ }^{131} \mathrm{I}$ treatment was accepted before, v) no other malignant tumor existed except PTC, and vi) tumor tissue samples were achievable. All patients were prepared with at least 3 weeks L-T4 withdrawal and 1 month low-iodine diet according to the guideline of Chinese Medical Association (CMA) before the ${ }^{131}$ I treatment. ${ }^{18}$ Before ${ }^{131}$ I treatment, TSH, TSHstimulated $\mathrm{Tg}$ ( $\mathrm{sTg}$ ) and $\mathrm{TgAb}$ in blood were measured. Neck ultrasonography, neck and chest CT scans were performed as well, at the same time. The postoperative tumor tissue samples were collected, and blood samples were taken the day before ${ }^{131}$ I treatment. Gene mutation in ctDNA and tumor tissue was detected using nextgeneration-sequencing (NGS) technology. Pathological and clinical data of the patients were collected from medical documents. According to the guidelines of CMA, therapeutic ${ }^{131} \mathrm{I}$ dose was determined as follow: i) 3.7 $\mathrm{GBq}$ for routine ablation of residual thyroid tissue; ii) lower dose if massive functional thyroid remnant could be confirmed in ${ }^{99} \mathrm{TcO}_{4}{ }^{-}$thyroid scan to alleviate local radiation inflammation; iii) higher dose if a high sTg level was found to eradicate potential malignant lesions (either local tumor or metastasis) despite whether definite lesion could be confirmed or not because a high sTg level generally implies the existence of malignancy. Post-treatment whole-body ${ }^{131} \mathrm{I}$ scan $\left({ }^{131} \mathrm{I} \mathrm{Rx}-\mathrm{WBS}\right)$ in the anterior and posterior projections were obtained 4 days after ${ }^{131}$ I treatment. This study was approved by the Medical Research Ethics Committee of Hangzhou Cancer Hospital and all patients signed informed consent form. After the initial radioiodine therapy, all patients were regularly followed-up with Tg, TgAb, TSH, neck ultrasonography and chest CT if necessary. The outcome of ${ }^{131} \mathrm{I}$ treatment was assessed 6 months later with the same preparation as before. ${ }^{131}$ I diagnostic whole-body scan (Dx-WBS) was performed 3 days after $111 \mathrm{MBq}{ }^{131} \mathrm{I}$ administration. Clinical cure was defined as fulfilling all the following criteria: i) no abnormal accumulation on ${ }^{131} \mathrm{I}$ Dx-WBS; ii) $\mathrm{sTg}<1 \mathrm{ng} / \mathrm{mL}$ or $\mathrm{Tg}<0.1 \mathrm{ng} / \mathrm{mL}$ in the absence of $\mathrm{TgAb}$; iii) no evidence of disease on neck ultrasonography and chest CT. The patients who achieved clinical cure were followed-up continuously if without recurrence of the disease.

\section{Sample Collection and DNA Extraction}

$10 \mathrm{~mL}$ peripheral blood was collected in Streck cfDNA BCT tube before ${ }^{131}$ I treatment for all the patients. 
Then the tubes were turned upside down gently for mixing, at least 10 times, and stored at $6-25^{\circ} \mathrm{C}$ until use (within 3 days). For tissue specimens, the total mass of each specimen should be no less than $60 \mathrm{mg}$, the proportion of tumor cells should be no less than $70 \%$, and the proportion of necrotic cells should be no more than $10 \%$. Tissue samples were stored in DNA preservation tubes (1Gene, Hangzhou, China) and handled in 2 days.

DNeasy Blood \& Tissue Kit (Qiagen, Hilden, Germany) was used strictly according to manufacturer's instructions to extract cfDNA (cell free DNA) and gDNA (genomic DNA) from blood samples and tissue samples respectively. The integrity, purity, and concentration of extracted DNA were measured using DNA gel electrophoresis, nanodrop, and Qubit respectively. The extracted DNA with an OD ratio between 1.8 to 2.0 and a mass more than $10 \mathrm{ng}$ was considered qualified and would be used for the following library construction.

\section{Library Construction and Target-Capture Sequencing}

DNA library was constructed according to the manufacturer's instructions of KAPA Hyperprep kit (Illumina Inc., San Diego, USA). Briefly, the steps included DNA fragmenting, terminal repair, 3'-end dAtailing adenylation, and ligation to indexed adapters. The quality and size of prepped library were evaluated by real-time PCR testing and 2100 Bioanalyzer (Agilent Inc., California, USA) respectively. A panel which covers 66 cancer-related genes (1Gene Inc., Hangzhou, China) was used in the hybrid-capture enrichment procedure, and the following PE150 sequencing (pair-end 150bp) was performed on the CN500 platform (Illumina Inc., San Diego, USA). The average sequencing depth of the target region of blood samples and tissue samples was $1637 \mathrm{x}$ and 866x respectively.

\section{Preliminary Analysis and Variant Calling}

The pre-alignment quality control of sequencing data was performed with FastQC. The quality recalibration, such as the removing of duplication reads, low mapping reads and adaptor sequences, was performed with GATK and Picard. Then the reads were aligned to human reference genome hg19 with BWA. Putative somatic variants were called with SpeedSeq and VarScan2 and the identified variants were annotated with ANNOVA. We excluded variants presented in highly repetitive regions, and also excluded common SNPs with minor allele frequency of $>0.001$ as recorded in 1000 genomes. Databases such as dbSNP, ClinVar, Cosmic were applied for variants' filtering, and integrated mutation prediction software such as Polyphen, SIFT were used to analyze the identified variants.

\section{Statistical Analysis}

The relationship of $B R A F$ mutation with the outcome of the first postoperative ${ }^{131}$ I treatment and malignant biological characteristics were analyzed by Fisher's exact test. All statistical analyses were performed by STATA 14.0. A $P$ value $<0.05$ was considered statistically significant.

\section{Results}

After screening, finally, a total of 33 patients ( 8 males, 25 females) were enrolled in the study as shown in Figure 1. The mean age at ${ }^{131} \mathrm{I}$ treatment was 44.8 \pm 15.4 years (range, $11-70$ years). The pathological characteristics of the patients were summarized in Table 1. The outcome of ${ }^{131}$ I treatment was assessed 6 months later and 9 patients were lost during the follow-up. Clinical cure was achieved in 19 patients (79.2\%, 19/ 24). $B R A F$ mutation was detected in 25 patients $(75.8 \%$, $25 / 33$ ) and 5 patients $(15.2 \%, 5 / 33)$ in tumor tissues and ctDNA respectively, as summarized in Table 2. By the status of $B R A F$ mutation, the patients were divided into 2 groups: 1) $B R A F$ mutation; 2) $B R A F$ wild-type, for both tissue and ctDNA analysis. In each category of ctDNA and tumor tissue, the outcome of the first ${ }^{131} \mathrm{I}$ treatment and the malignant characteristics showed no significant relationship with $B R A F$ mutation $(\mathrm{P} \geq 0.05)$, as presented in Tables 3 and 4.

\section{Discussion}

In recent years, gene mutations in thyroid carcinoma have been intensively investigated, and most of them were tumor tissue based. In PTC, $B R A F$ mutation has the highest incidence. According to the studies in Chinese population, the incidence of $B R A F$ mutation ranged from $59 \%$ to $72.4 \%$ in PTC patients. ${ }^{19-21}$ Similar to those findings, the incidence of $B R A F$ mutation in tumor tissue in our patients was $75.8 \%$. 


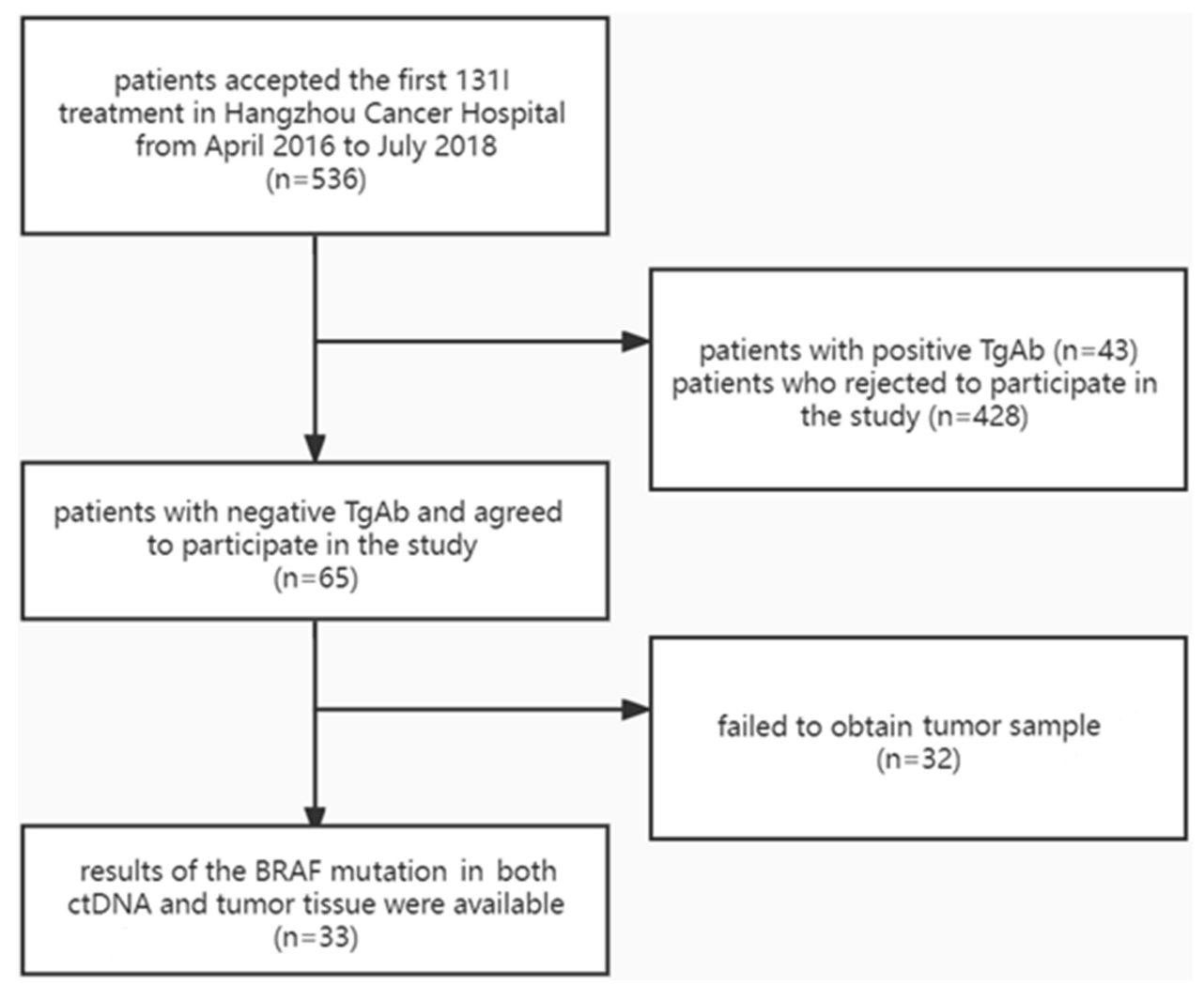

Figure I The flow chart of patient inclusion.

However, the incidence of $B R A F$ mutation in ctDNA was only $15.2 \%$. We speculate that the low incidence of $B R A F$ mutation in ctDNA might be attributed to the

Table I Pathological Characteristics of Patients $(n=33)$

\begin{tabular}{|c|c|c|}
\hline Characteristic & Number & Percentage (\%) \\
\hline \multicolumn{3}{|l|}{$\begin{array}{l}\text { Primary tumor size (largest } \\
\text { diameter) }\end{array}$} \\
\hline$\leq \mathrm{Icm}$ & 6 & 18.2 \\
\hline$>\mathrm{Icm}$ & 27 & 81.8 \\
\hline \multicolumn{3}{|l|}{ Number of primary tumor lesions } \\
\hline Single lesion & 9 & 27.3 \\
\hline Multiple lesions & 24 & 72.7 \\
\hline \multicolumn{3}{|l|}{ Number of metastatic lymph nodes } \\
\hline$<5$ & 15 & 45.5 \\
\hline$\geq 5$ & 18 & 54.5 \\
\hline \multicolumn{3}{|l|}{ Extrathyroidal extension } \\
\hline Yes & 20 & 60.6 \\
\hline $\begin{array}{l}\text { Extension into sternothyroid } \\
\text { muscle or perithyroidal soft } \\
\text { tissues }\end{array}$ & 18 & \\
\hline Lung or bone & 2 & \\
\hline No & 13 & 39.4 \\
\hline
\end{tabular}

following. First, tumor cells in certain patients might be confined to the tumor sites without involvement of the blood vessels. Second, although tumor cells might possibly enter the blood at the time of surgery, the amount of the tumor cells might be small due to the complete removal of the lesions, including primary tumor and local lymph nodes metastases in a portion of the patients. Third, before ${ }^{131} \mathrm{I}$ treatment, usually a preparation period of at least one month is needed to elevate TSH level by L-T4 withdrawal and restricted iodine intake after the surgery. In this period, the amount of tumor cells might further decrease. As a consequence, gene mutation in ctDNA might not be detectable at the time of blood sampling in some patients. Pupilli et al reported a similar finding, that $71 \%$ of patients with PTC originally had $B R A F$ mutation in ctDNA but became BRAF mutation free after surgery. ${ }^{22}$ Post-operative ${ }^{131}$ I treatment is the most crucial adjuvant therapy that can significantly decrease the recurrence of PTC with medium or high risk after thyroidectomy, to obtain a better DFS. ${ }^{23}$ The ablation of residual thyroid tissue and the elimination of potentially unresectable ${ }^{131} \mathrm{I}$-avid metastasis by ${ }^{131} \mathrm{I}$ treatment could facilitate both follow-up using $\mathrm{Tg}$ and ${ }^{131} \mathrm{I}$ uptake test 


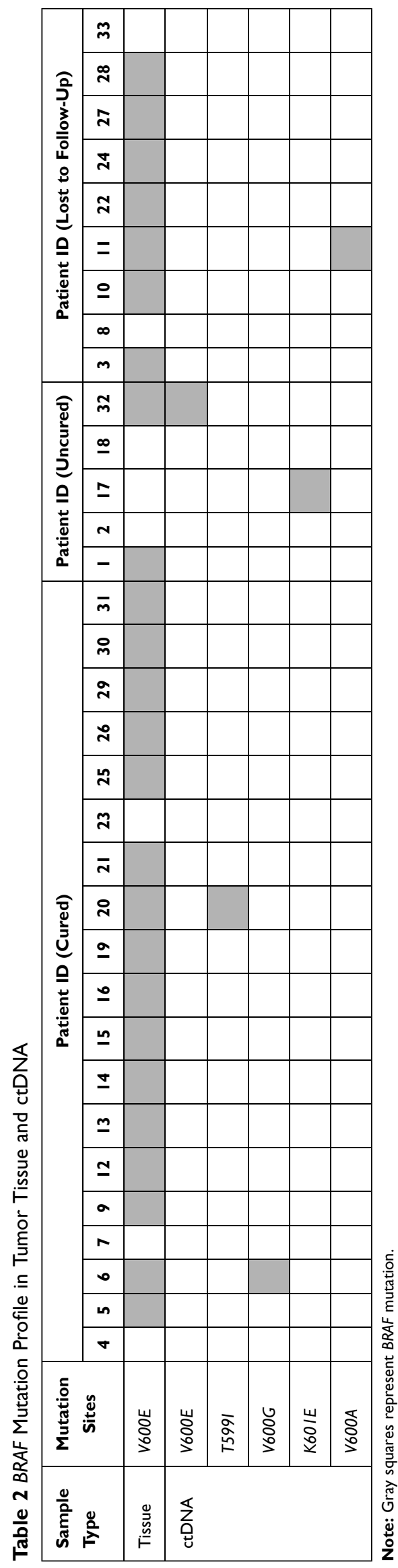

and possible further therapy, ie, next ${ }^{131} \mathrm{I}$ treatment. A number of studies have found that BRAF mutation was significantly related to the down-regulation of the expression of iodide-metabolism related gene and protein, eg, sodium-iodide symporter (NIS). ${ }^{24-26}$ Yang et al found that in PTC patients with distant metastasis, the incidence of non-iodine avid foci was $84.2 \%$ in patients with $B R A F$ mutation, while this incidence was only $5.6 \%$ in patients with wild-type $B R A F .{ }^{27}$ However, as for the relationship between $B R A F$ mutation and the outcome of ${ }^{131}$ I treatment, no common consensus has been achieved so far. In 2015 ATA guidelines, for the first time, molecular markers were introduced in the stratification of the recurrence risk of thyroid cancer. However, no recommendation was given concerning the relationship between $B R A F$ mutations and postoperative recurrence in PTC due to the inconsistent findings in literature. A study based on the data of 15 years follow-up demonstrated that BRAF mutation is an independent risk factor for the recurrence of $\mathrm{PTC}^{7}$ Sato et al indicated that the fractional abundance of mutated $B R A F^{V 600 E}$ in post-surgery ctDNA might predict PTC recurrence. ${ }^{9}$ On the contrary, other studies reported that $B R A F$ mutation may have no influence on the outcome of ${ }^{131}$ I treatment in PTC patients. ${ }^{19,28}$ In our study, $B R A F$ mutation was detected in both tumor tissue and ctDNA in 1 patient with lung and bone metastases and 3 patients without distant metastasis. However, compared with the patients without distant metastasis, the patient with distant metastases showed a higher titer of $B R A F$ mutation in ctDNA. The results implied that ctDNA might be a useful indicator for treatment decision in patients with distant metastases. However, since there was only one patient with distant metastases in our study, more investigations are needed to verify this finding.

The relationship of $B R A F$ mutation with malignant characteristics of PTC has not been fully understood. ${ }^{6,8,28,29}$ In accordance with previous studies, in our study, none of the characteristics such as age, gender, primary tumor size, multifocality of tumors, extrathyroidal extension, and lymph node metastasis showed significant relationship with $B R A F$ mutation in both tumor tissue and ctDNA, implying that $B R A F$ might be less relevant to the malignant biological characteristics of PTC. We detected multiple BRAF mutation sites in 1 patient in ctDNA. However, due to the small sample size, the clinical significance of 
Table 3 Relationship of BRAF Mutation in ctDNA with the Outcome of First ${ }^{131} \mathrm{I}$ Treatment and Other Characteristics in the Patients $(n=33)$

\begin{tabular}{|c|c|c|c|c|}
\hline & \multicolumn{3}{|c|}{ BRAF } & \multirow[t]{2}{*}{$P$ value } \\
\hline & Number & Mutation (n) & Wild-Type (n) & \\
\hline \multicolumn{5}{|l|}{ Age } \\
\hline$<55$ & 25 & 4 & 21 & 1.00 \\
\hline$\geq 55$ & 8 & I & 7 & \\
\hline \multicolumn{5}{|l|}{ Gender } \\
\hline Male & 8 & 0 & 8 & 1.00 \\
\hline Female & 25 & 5 & 20 & \\
\hline \multicolumn{5}{|c|}{ Outcome of ${ }^{|3|} \mid$ treatment $^{a}$} \\
\hline Cure & 19 & 2 & 17 & 0.18 \\
\hline Non-cure & 5 & 2 & 3 & \\
\hline \multicolumn{5}{|c|}{ Primary tumor size (largest diameter) } \\
\hline$\leq \mathrm{Icm}$ & 6 & 2 & 4 & 0.22 \\
\hline$>\mathrm{Icm}$ & 27 & 3 & 24 & \\
\hline \multicolumn{5}{|c|}{ Number of primary tumor lesions } \\
\hline Single lesion & 9 & I & 8 & 1.00 \\
\hline Multiple lesions & 24 & 4 & 20 & \\
\hline \multicolumn{5}{|c|}{ Number of metastatic lymph nodes } \\
\hline$<5$ & 15 & 3 & 12 & 0.63 \\
\hline$\geq 5$ & 18 & 2 & 16 & \\
\hline \multicolumn{5}{|c|}{ Extrathyroidal extension } \\
\hline Yes & 20 & 5 & 15 & 0.13 \\
\hline No & 13 & 0 & 13 & \\
\hline
\end{tabular}

Note: ${ }^{\text {a9 }}$ patients were lost in follow-up.

multiple $B R A F$ mutation sites still needs to be elucidated.

Other than $B R A F$ gene, a number of gene mutations such as TERT, PTEN, PIK3CA, TP53, RAS have also been studied in the origination and malignant progression of thyroid cancer. It has been noticed that the co-existence of $B R A F$ mutation and other mutations might be involved in the tumorigenesis and dedifferentiation of PTC and may be more predictive of prognosis. Xing et al reported that the combination of BRAF mutation and TERT promoter mutation in PTC was the most aggressive mutation type and had the highest incidence of recurrence, compared with single $B R A F$ mutation. ${ }^{30}$ In our study, only $B R A F$ gene mutation was studied. To overcome this disadvantage, further investigations are demanded to clarify the influence and the interaction of more gene mutations in
PTC. Other limitations should also be noted in our preliminary study. First, the samples were not sufficient, especially for tumor tissue, due to the difficulty of sample collection from different hospitals; second, the sensitivity of our sequencing platform for ctDNA detection was relatively low (1637X), we may have failed to detect the mutation in patients with trace amount of mutation. Our subsequent study will enroll more samples for both ctDNA and tumor tissue. In addition, more gene mutations will be investigated as the targets, along with expanding the depth of sequencing and improving sequencing sensitivity.

In conclusion, the value of $B R A F$ mutation alone might be limited in predicting the therapeutic outcome of first ${ }^{131} \mathrm{I}$ treatment in PTC. No certain relevance was found between $B R A F$ mutation and malignant biological features in PTC. 
Table 4 Relationship of BRAF Mutation in Tumor Tissues with the Outcome of First ${ }^{|3|} \mid$ Treatment and Other Characteristics in the Patients $(n=33)$

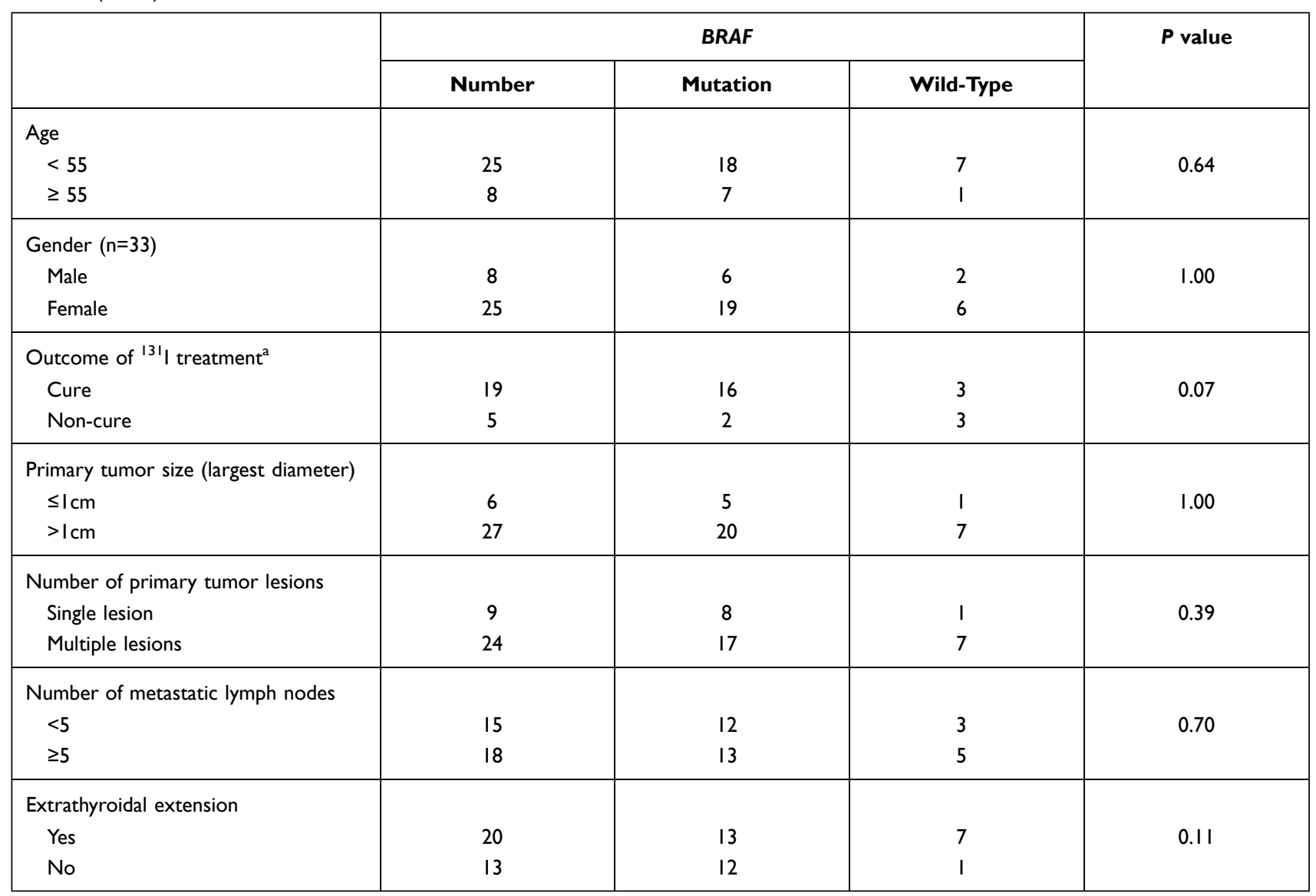

Note: ${ }^{a} 9$ patients were lost in follow-up.

\section{Data Sharing Statement}

The datasets used and/or analyzed during the present study are available from the corresponding author, Chunlei Zhao, upon reasonable request.

\section{Ethics Approval}

The present study was approved by the Ethics Committee of the Hangzhou Cancer Hospital.

\section{Consent to Participate}

Informed consent was obtained from all individual participants included in the study.

\section{Consent for Publication}

Patients signed informed consent regarding publishing their data and photographs.

\section{Author Contributions}

All authors made a significant contribution to the work reported, whether that is in the conception, study design, execution, acquisition of data, analysis and interpretation, or in all these areas; all authors took part in drafting, revising or critically reviewing the article; all authors gave final approval of the version to be published; all authors have agreed on the journal to which the article has been submitted and agree to be accountable for all aspects of the work.

\section{Funding}

This study was sponsored by the Key Project of Science and Technology Planning of Health in Hangzhou (2018ZD02), the project of public beneficial technology research and social development of Zhejiang Province (LGF20H180010), and the Key project of Health Commission in Hangzhou (OO20190490). This study was conducted in accordance with the Declaration of Helsinki.

\section{Disclosure}

All authors have no conflicts of interest to declare that are relevant to the content of this article. 


\section{References}

1. Fagin JA, Wells SA Jr. Biologic and clinical perspectives on thyroid cancer. $N$ Engl $J$ Med. 2016;375(11):1054-1067. doi:10.1056/ NEJMra1501993

2. Celik M, Bulbul BY, Ayturk S, et al. The relation between BRAFV600E mutation and clinicopathological characteristics of papillary thyroid cancer. Med Glas. 2020;17(1):30-34.

3. Abdullah MI, Junit SM, Ng KL, Jayapalan JJ, Karikalan B, Hashim OH. Papillary thyroid cancer: genetic alterations and molecular biomarker investigations. Int J Med Sci. 2019;16(3):450-460. doi:10.7150/ijms.29935

4. Xing M. BRAF mutation in thyroid cancer. Endocr Relat Cancer. 2005;12(2):245-262. doi:10.1677/erc. 1.0978

5. Xing M, Alzahrani AS, Carson KA, et al. Association between BRAF V600E mutation and recurrence of papillary thyroid cancer. $J$ Clin Oncol. 2015;33(1):42-50. doi:10.1200/JCO.2014.56.8253

6. Elisei R, Viola D, Torregrossa L, et al. The BRAF(V600E) mutation is an independent, poor prognostic factor for the outcome of patients with low-risk intrathyroid papillary thyroid carcinoma: single-institution results from a large cohort study. J Clin Endocrinol Metab. 2012;97 (12):4390-4398. doi:10.1210/jc.2012-1775

7. Elisei R, Ugolini C, Viola D, et al. BRAF(V600E) mutation and outcome of patients with papillary thyroid carcinoma: a 15-year median follow-up study. $J$ Clin Endocrinol Metab. 2008;93 (10):3943-3949. doi:10.1210/jc.2008-0607

8. Xing M, Westra WH, Tufano RP, et al. BRAF mutation predicts a poorer clinical prognosis for papillary thyroid cancer. J Clin Endocrinol Metab. 2005;90(12):6373-6379. doi:10.1210/jc.2005-0987

9. Sato A, Tanabe M, Tsuboi Y, et al. Circulating tumor DNA harboring the BRAF V600E mutation may predict poor outcomes of primary papillary thyroid cancer patients. Thyroid. 2021. doi:10.1089/thy.2021.0267

10. Enumah S, Fingeret A, Parangi S, Dias-Santagata D, Sadow PM, Lubitz CC. BRAF(V600E) mutation is associated with an increased risk of papillary thyroid cancer recurrence. World J Surg. 2020;44 (8):2685-2691. doi:10.1007/s00268-020-05521-2

11. Yao J, Zang W, Ge Y, et al. RAS/BRAF circulating tumor DNA mutations as a predictor of response to first-line chemotherapy in metastatic colorectal cancer patients. Can J Gastroenterol Hepatol. 2018;2018:4248971. doi:10.1155/2018/4248971

12. McCoach CE, Blakely CM, Banks KC, et al. Clinical utility of cell-free DNA for the detection of ALK fusions and genomic mechanisms of ALK inhibitor resistance in non-small cell lung cancer. Clin Cancer Res. 2018;24(12):2758-2770. doi:10.1158/ 1078-0432.CCR-17-2588

13. Ma F, Zhu W, Guan Y, et al. ctDNA dynamics: a novel indicator to track resistance in metastatic breast cancer treated with anti-HER2 therapy. Oncotarget. 2016;7(40):66020-66031. doi:10.18632/oncotarget.11791

14. Yong E. Cancer biomarkers: written in blood. Nature. 2014;511 (7511):524-526. doi:10.1038/511524a

15. Bettegowda C, Sausen M, Leary RJ, et al. Detection of circulating tumor DNA in early- and late-stage human malignancies. Sci Transl Med. 2014;6(224):224ra24. doi:10.1126/scitranslmed.3007094

16. Qin Y, Wang JR, Wang Y, et al. Clinical utility of circulating cell-free DNA mutations in anaplastic thyroid carcinoma. Thyroid. 2021;31 (8):1235-1243. doi:10.1089/thy.2020.0296

17. Haugen BR, Alexander EK, Bible KC, et al. 2015 American thyroid association management guidelines for adult patients with thyroid nodules and differentiated thyroid cancer: the American thyroid association guidelines task force on thyroid nodules and differentiated thyroid cancer. Thyroid. 2016;26(1):1-133.
18. Endocrinology Chinese Society of, Association Endocrine Group of Surgery Branch of Chinese Medical, Association Committee for Head and neck Oncology of Chinese AntiCancer, Medicine Chinese Society of Nuclear. Management guidelines for the diagnosis and management of thyroid nodules and differentiated thyroid cancer. Chin J Nucl Med Mol Imaging. 2013;33(2):96-115.

19. Shen G, Kou Y, Liu B, Huang R, Kuang A. BRAFV600E mutation does not significantly affect the efficacy of radioiodine therapy in patients with papillary thyroid carcinoma without known distant metastases. Clin Nucl Med. 2018;43(7):e215-e219. doi:10.1097/ RLU.000000000002142

20. Liang J, Cai W, Feng D, et al. Genetic landscape of papillary thyroid carcinoma in the Chinese population. J Pathol. 2018;244(2):215-226. doi:10.1002/path.5005

21. Pan W, Zhou L, Ge M, et al. Whole exome sequencing identifies IncRNA GAS8-AS1 and LPAR4 as novel papillary thyroid carcinoma driver alternations. Hum Mol Genet. 2016;25(9):1875-1884. doi:10.1093/hmg/ddw056

22. Pupilli C, Pinzani P, Salvianti F, et al. Circulating BRAFV600E in the diagnosis and follow-up of differentiated papillary thyroid carcinoma. $J$ Clin Endocrinol Metab. 2013;98(8):3359-3365. doi:10.1210/ jc. 2013-1072

23. Benbassat CA, Mechlis-Frish S, Hirsch D. Clinicopathological characteristics and long-term outcome in patients with distant metastases from differentiated thyroid cancer. World $J$ Surg. 2006;30 (6):1088-1095. doi:10.1007/s00268-005-0472-4

24. Bastos AU, Oler G, Nozima BH, Moyses RA, Cerutti JM. BRAF V600E and decreased NIS and TPO expression are associated with aggressiveness of a subgroup of papillary thyroid microcarcinoma. Eur J Endocrinol. 2015;173(4):525-540. doi:10. 1530/EJE-15-0254

25. Spitzweg C, Bible KC, Hofbauer LC, Morris JC. Advanced radioiodine-refractory differentiated thyroid cancer: the sodium iodide symporter and other emerging therapeutic targets. Lancet Diabetes Endocrinol. 2014;2(10):830-842. doi:10.1016/S22138587(14)70051-8

26. Chakravarty D, Santos E, Ryder M, et al. Small-molecule MAPK inhibitors restore radioiodine incorporation in mouse thyroid cancers with conditional BRAF activation. J Clin Invest. 2011;121(12):47 00-4711. doi:10.1172/JCI46382

27. Yang K, Wang H, Liang Z, Liang J, Li F, Lin Y. BRAFV600E mutation associated with non- radioiodine-avid status in distant metastatic papillary thyroid carcinoma. Clin Nucl Med. 2014;39(8):6 75-679.

28. Barbaro D, Incensati RM, Materazzi G, et al. The BRAF V600E mutation in papillary thyroid cancer with positive or suspected pre-surgical cytological finding is not associated with advanced stages or worse prognosis. Endocrine. 2014;45(3):462-468. doi:10.1007/s12020-013-0029-5

29. Tufano RP, Teixeira GV, Bishop J, Carson KA, Xing M. BRAF mutation in papillary thyroid cancer and its value in tailoring initial treatment: a systematic review and meta-analysis. Medicine (Baltimore). 2012;91(5):274-286. doi:10.1097/MD.0b013e31826a $9 \mathrm{c} 71$

30. Xing M, Liu R, Liu X, et al. BRAF V600E and TERT promoter mutations cooperatively identify the most aggressive papillary thyroid cancer with highest recurrence. J Clin Oncol. 2014;32 (25):2718-2726. doi:10.1200/JCO.2014.55.5094 


\section{Publish your work in this journal}

The International Journal of General Medicine is an international, peer-reviewed open-access journal that focuses on general and internal medicine, pathogenesis, epidemiology, diagnosis, monitoring and treatment protocols. The journal is characterized by the rapid reporting of reviews, original research and clinical studies across all disease areas. The manuscript management system is completely online and includes a very quick and fair peer-review system, which is all easy to use. Visit http://www.dovepress.com/ testimonials.php to read real quotes from published authors.

Submit your manuscript here: https://www.dovepress.com/international-journal-of-general-medicine-journal 